\title{
Nonlinear Model Predictive Control with Terminal Invariant Manifolds for Stabilization of Underactuated Surface Vessel
}

\author{
Lutao Liu, ${ }^{1}$ Zhilin Liu, ${ }^{2}$ and Jun Zhang $^{3}$ \\ ${ }^{1}$ College of Information and Telecommunication, Harbin Engineering University, Harbin 150001, China \\ ${ }^{2}$ College of Automation, Harbin Engineering University, Harbin 150001, China \\ ${ }^{3}$ School of Electrical and Information Engineering, Jiangsu University, Zhenjiang, Jiangsu 212013, China \\ Correspondence should be addressed to Zhilin Liu; liuzhilin@hrbeu.edu.cn
}

Received 21 September 2013; Accepted 14 October 2013

Academic Editor: Xiaojie Su

Copyright (C) 2013 Lutao Liu et al. This is an open access article distributed under the Creative Commons Attribution License, which permits unrestricted use, distribution, and reproduction in any medium, provided the original work is properly cited.

A nonlinear model predictive control (MPC) is proposed for underactuated surface vessel (USV) with constrained invariant manifolds. Aimed at the special structure of USV, the invariant manifold under the given controller is constructed in terms of diffeomorphism and Lyapunov stability theory. Based on MPC, the states of the USV are steered into the constrained terminal invariant manifolds. After the terminal manifolds set is reached, a linear feedback control is used to stabilize the system. The simulation results verified the effectiveness of the proposed method. It is shown that, based on invariant manifolds constraints, it is easy to get the MPC for the USV and it is suitable for practical application.

\section{Introduction}

The waterjet propulsor is widely used to thrust existing planning surface vessels. The conventional method to control planning surface vessels is indirectly achieved through the course control which is actuated by a steerable nozzle. If the planar position and course are controlled directly, we need to regulate the angle and the thrust force of the steering nozzle to control the movements in three degrees of freedom synchronously. Obviously, the control system of planning surface vessels is typical underactuated system. In order to ensure the safety of the planning surface vessel, many constraints such as the angle of the steering nozzle and radius of gyration must be in consideration in the design process of ship motion controller; otherwise it will undermine the performance of the planning surface vessels and even lead to collapse of the hull and other serious consequences. The underactuated system with constraints is essentially nonlinear system and cannot be stabilized by any smooth time-invariant control laws. Predictive control is an effective optimization control method to deal with constrains [1-6].

In general, the use of MPC in an underactuated structure system necessitates a means of switching among the available models to the one that best describes the current operating condition. A closely related work is the stability analysis of switched stochastic systems by [7] in which dissipativitybased sliding mode control was constructed. Since designing MPC controllers that stabilize underactuated system may not result in a stable global closed-loop system, closed-loop stability in switch model/control approaches has also been studied [8]. The study in the paper is aimed to analyse the underactuated characteristics of planning surface vessels and guarantee safe movements of the vessels. Furthermore, based on predictive control approach, the problem of the stabilization and tracking control is solved under the condition of reserved safety constraints.

Control of underactuated systems has been one of the active research topics due to its intrinsic nonlinear nature and practical applications. As a typical example of underactuated systems, control of an underactuated ship has been focused on recently. The main difficulty in the control of underactuated ships is that they are not actuated in the sway axis. This configuration is the most common among the surface ships [9]. Furthermore, unlike underactuated systems with nonintegrable constraints, the surface vessels under the consideration are a class of underactuated systems 
with nonintegrable dynamics and are not transformed into a driftless system [10]. Nevertheless, several authors have studied the trajectory tracking control problem. A discontinuous control approach with two stage control laws switched on at given time is proposed based on the stability analysis of the global transformed system in [11]. In [12], the authors proposed a kinematic tracking controller that achieved global exponential practical stability of an underactuated surface vessel. In [13], a continuous time-invariant control law was proposed to obtain semiglobal exponential position and orientation tracking, provided the desired angular trajectory remains positive. Based on the cascaded approach, a global tracking result was obtained in $[14,15]$. The stability analysis relied on the stability theory of linear time-varying systems. An application of the recursive technique proposed in [16] for the standard chain form systems was used in [17] to provide exponential stability of the reference trajectory. Based on Lyapunov's direct method and passivity approach, two constructive tracking solutions were proposed in [18] for an underactuated ship. The constructive control design procedure exploited the inherent cascade-interconnected structure of the ship dynamics and actually generated an explicit Lyapunov function whose availability might suit the requirements of robust and adaptive control design. With the help of the backstepping design methodology, a nonlinear time-invariant control law was proposed in [19] for an underactuated surface vessel. But the control design in [19] was not complete and lacked a further step in the backstepping procedure. In the comment letter [20], the control laws in [19] were revised and the states decayed asymptotically to zero.

Model predictive control (MPC) is a popular technique for the control of slow dynamical system subject to input and state constraints. At any time instant, MPC requires the online solution of an optimization problem to compute optimal control inputs over a fixed number of future time instants, known as the finite horizon or quasi-infinite horizon. Using MPC, it is possible to handle inequality constraints on the manipulated and controlled variables in a systematic manner during the design and implementation of the controller. Perhaps the principal shortcoming of existing MPC-base control techniques is their inability to explicitly incorporate plant model uncertainty. The fact that the rudder actuation is limited in amplitude and rate makes MPC approach a natural choice for the design of the path following controller. In [21], a standard model predictive control approach for path following with roll constraints of marine surface vessels in calm water using the rudder as the control input has been proposed. The focus is on satisfying all the input (rudder) and state (roll) constraints while achieving satisfactory path following performance. For notational convenience the ship dynamics in [21] are written into linearized matrix form based on the assumption that surge velocity is constant and the yaw moment is proportional to the rudder angle. However, the former assumption is hardly admissible in engineering. In [22], an analytic model predictive controller is presented for path following of an underactuated ship maneuvering along a predefined path. The mathematical model of ship motion is described by using

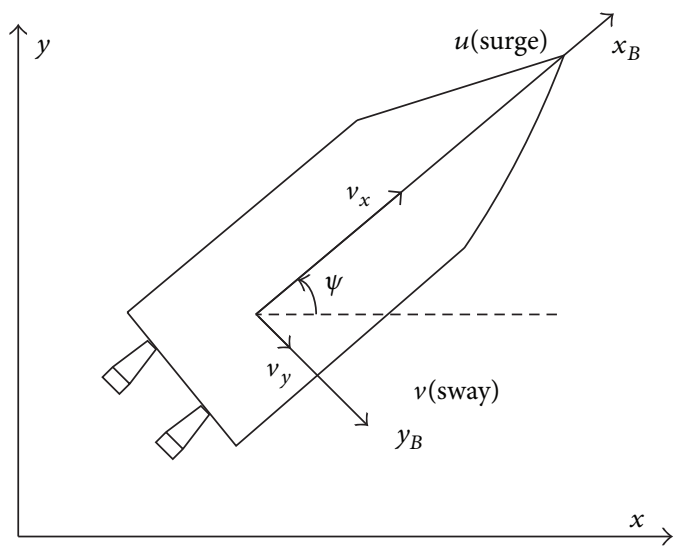

FIGURE 1: Underactuated surface vessel.

Serret-Frenet frame, and a systematic method is provided to guarantee the stability of the closed-loop system in terms of transforming the original single-input multiple-output (SIMO) into an equivalent single-input single-output (SISO) system. However, the MPC algorithm proposed in [22] is designed as an analytic model predictive controller which is affected by the accuracy of the parameters.

This paper illuminates the stabilization approach for underactuated surface vessels with only a surge force and a yaw moment. A nonlinear model predictive controller is presented for steering the states of underactuated ship into a desired terminal invariant manifolds. After the terminal manifolds set is reached, a linear feedback control is used to stabilize the system. However, in the techniques mentioned above, plant modeling is the critical step to obtain feedback controller and the control result is strongly influenced by the model; the problems inherent to plant modeling are inevitable. Moreover, even complex models cannot cover all the system dynamics [23-26]. In the future work, we will make efforts to design data-driven MPC controllers to overcome these problems.

\section{Problem Formulation}

In this paper, we consider the trajectory tracking control problem of a surface vessel shown in Figure 1. There is no side thruster, but two independent main thrusters are located at a distance from the center line in order to provide both surge force and yaw moment.

The dynamics and kinematics of an underactuated surface vessel are described as follows [27]:

$$
\begin{gathered}
M \dot{\nu}+C(\nu) \nu+D \nu=\tau, \\
\dot{\eta}=J(\eta) \nu .
\end{gathered}
$$

The inertia matrix $M=\operatorname{diag}\left\{m_{11}, m_{22}, m_{33}\right\}$ and the damping matrix $D=\operatorname{diag}\left\{d_{11}, d_{22}, d_{33}\right\}$ are constant and positive definite. The vector $\tau=\left[\tau_{1}, \tau_{2}, \tau_{3}\right]$ denotes the control forces in surge and sway and control torque in yaw. In this paper, the surface vessel is assumed as the common thruster configuration that has no side thruster, such as $\tau_{2}=0$. So 
the second component of (1) behaves as a nonholonomic constraint, which is a nonintegrable relation involving not only the generalized coordinates and velocities but also the generalized accelerations [28]. $C(\nu)$ is the matrix of Coriolis and centripetal terms also including added mass. $J(\eta)$ is the rotation matrix for the transformation between body-fixed and earth-fixed coordinates:

$$
\begin{gathered}
C(\nu)=\left[\begin{array}{ccc}
0 & 0 & -m_{22} v \\
0 & 0 & m_{11} u \\
m_{22} v & -m_{11} u & 0
\end{array}\right], \\
J(\eta)=\left[\begin{array}{ccc}
\cos (\psi) & -\sin (\psi) & 0 \\
\sin (\psi) & \cos (\psi) & 0 \\
0 & 0 & 1
\end{array}\right] .
\end{gathered}
$$

The vector $\eta=[x, y, \psi]^{T}$ denotes the North and East positions and orientation of the underactuated surface vessel in the earth-fixed coordinate system. The vector $v=[u, v, r]^{T}$ denotes the linear velocities in surge and sway and the angular velocity in yaw.

As a general accepted conclusion [29, 30], there is no continuous time-invariant feedback control law that makes the zero origin an asymptotically stable equilibrium of the system (1) and (2), for the system does not satisfy Brocketts condition [31]. Then time-varying and discontinuous control approaches are only taken into account in this paper.

Neglecting the motions in heave, roll, and pitch, the simplified kinematic model which describes the geometrical relationship between the earth-fixed (E-frame) and the bodyfixed (B-frame) motion is given as

$$
\begin{gathered}
m_{11} \dot{u}-m_{22} v r+d_{11} u=\tau_{1}, \\
m_{22} \dot{v}+m_{11} u r+d_{22} v=0, \\
m_{33} \dot{r}+\left(m_{22}-m_{11}\right) u v+d_{33} r=\tau_{3}, \\
\dot{x}=u \cos \psi-v \sin \psi, \\
\dot{y}=u \sin \psi+v \cos \psi, \\
\dot{\psi}=r .
\end{gathered}
$$

The following global coordinate transformation and feedback transformation are adopted before control design. Define

$$
\begin{gathered}
z_{1}=x \cos (\psi)+y \sin (\psi), \\
z_{2}=v, \\
z_{3}=-x \sin (\psi)+y \cos (\psi)+\frac{m_{22}}{d_{22} v}, \\
z_{4}=\psi, \\
z_{5}=-\frac{m_{11}}{d_{22} u}-z_{1}, \\
z_{6}=r .
\end{gathered}
$$

It is proved that the state transformation (5) is a global diffeomorphism [32]. The feedback transformation is

$$
\begin{gathered}
w_{1}=\left(\frac{d_{11}}{d_{22}}-1\right) u-z_{3} z_{6}-\frac{\tau_{1}}{d_{22}}, \\
w_{2}=\frac{\left(m_{11}-m_{22}\right) u v}{m_{33}}-\frac{d_{33} r}{m_{33}}+\frac{\tau_{3}}{m_{33}} .
\end{gathered}
$$

With the state and feedback transformation (5)-(6), the system (1)-(2) is eventually transformed to

$$
\begin{gathered}
\dot{z}_{1}=-\frac{d_{22}}{m_{11}} z_{1}-\frac{d_{22}}{m_{11}} z_{5}+z_{3} z_{6}-\frac{m_{22}}{d_{22}} z_{2} z_{6}, \\
\dot{z}_{2}=-\frac{d_{22}}{m_{22}} z_{2}+\frac{d_{22}}{m_{22}} z_{6}\left(z_{1}+z_{5}\right), \\
\dot{z}_{3}=z_{5} z_{6}, \quad \dot{z}_{4}=z_{6}, \\
\dot{z}_{5}=w_{1}, \quad \dot{z}_{6}=w_{2} .
\end{gathered}
$$

The system (7) has the same diffeomorphism properties as the system (1) and (2) [32]; that is, if $\lim _{t \rightarrow \infty} z_{i}=0(1 \leq i \leq 6)$ then $(x, y, \psi, u, v, r)$ converges to zero as $t \rightarrow \infty$.

Lemma 1. If there exists a control law which globally uniformly asymptotically stabilizes the system

$$
\begin{gathered}
\dot{z}_{3}=z_{5} z_{6}, \quad \dot{z}_{4}=z_{6}, \\
\dot{z}_{5}=w_{1}, \quad \dot{z}_{6}=w_{2},
\end{gathered}
$$

then the system (7) under the control law is also globally uniformly asymptotically stabilized.

Proof. An approach to prove Lemma 1 based on Lyapunov stability theorem has been given in [28]. Therefore, to stabilize the system (1) and (2), it is only needed to design a stabilizing control law for the system (8). In the following section, a discontinuous and time-varying control approach with MPC control method is proposed for the stabilization of the system (8).

\section{Control Design}

Let us recall the following definitions firstly.

Definition 2. Let $\Phi: R^{n} \rightarrow R^{p}$ be a smooth map. A manifold $M=\left\{x \in R^{n}: \Phi(x)=0\right\}$ is said to be invariant for the control system $\dot{x}=f(x, u)$ if all system trajectories starting in $M$ at $t=t_{0}$ remain in this manifold for all $t \geq t_{0}$. In other words, the Lie derivative of $\Phi$ along the vector field $f$ is zero $\left(L_{f} \Phi(x)=0\right)$ for all $x \in M$.

Definition 3. A manifold $M=\left\{x \in R^{n}: \Phi(x)=0\right\}$ is said to be asymptotically attractive in an open domain $\Omega$ of $R^{n}$ if, for all $t \in R_{+}$such that $x\left(t_{0}\right) \in \Omega, \lim _{x \rightarrow \infty} x(t) \in M$.

3.1. Construction of the Invariant Manifold. First, in order to construct the invariant manifold of the system (8), assume that $\left[\begin{array}{ll}w_{1} & w_{2}\end{array}\right]^{T}$ is a linear state feedback such that

$$
\begin{gathered}
w_{1}=-k_{1} z_{5} \quad\left(k_{1}>0\right), \\
w_{2}=-k_{2} z_{6}-k_{3} z_{5} \quad\left(k_{2}>0, k_{3}>0, k_{1} \neq k_{2}\right) .
\end{gathered}
$$

Substitute (9) into (8); we obtain

$$
\begin{gathered}
\dot{z}_{3}=z_{5} z_{6}, \quad \dot{z}_{4}=z_{6}, \\
\dot{z}_{5}=-k_{1} z_{5}, \quad \dot{z}_{6}=-k_{2} z_{6}-k_{3} z_{5} .
\end{gathered}
$$


The resulting closed-loop system (10) can be successively integrated to obtain

$$
\begin{gathered}
z_{5}(t)=z_{50} e^{-k_{1} t} \\
z_{6}(t)=z_{60} e^{-k_{2} t}+\frac{k_{3} z_{50}}{k_{1}-k_{2}}\left(e^{-k_{1} t}-e^{-k_{2} t}\right), \\
z_{4}(t)=s_{4}\left(z_{0}\right)+\left[\frac{k_{3} z_{50}}{k_{2}\left(k_{1}-k_{2}\right)}-\frac{z_{60}}{k_{2}}\right] e^{-k_{2} t} \\
-\frac{k_{3} z_{50}}{k_{1}\left(k_{1}-k_{2}\right)} e^{-k_{1} t}, \\
z_{3}(t)=s_{3}\left(z_{0}\right)-\frac{1}{k_{1}+k_{2}}\left(z_{40} z_{60}-\frac{k_{3} z_{50}^{2}}{k_{1}-k_{2}}\right) e^{-\left(k_{1}+k_{2}\right) t} \\
-\frac{k_{3} z_{50}^{2}}{2 k_{1}\left(k_{1}-k_{2}\right)} e^{-2 k_{1} t},
\end{gathered}
$$

where $k_{1}, k_{2}, k_{3}, s_{3}\left(z_{0}\right)$, and $s_{4}\left(z_{0}\right)$ are the integration constants, which can be determined, at $t=0$, as a function of the initial conditions $z_{40}, z_{50}$, and $z_{60}$. Besides, from (11), one can

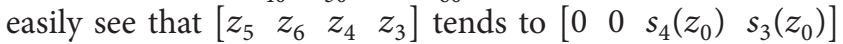
when $t$ tends to infinity. So, if we take the initial conditions such that $s_{4}\left(z_{0}\right)=0$ and $s_{3}\left(z_{0}\right)=0$, then the whole state tends to the origin. Setting $t=0$ in (11), $s_{4}(z)$ and $s_{3}(z)$ can be determined. Substituting $z_{0}$ by $z$ in the previous functions leads to

$$
\begin{gathered}
S_{4}(z)=z_{4}-\frac{k_{3} z_{5}}{k_{2}\left(k_{1}-k_{2}\right)}+\frac{z_{6}}{k_{2}}+\frac{k_{3} z_{5}}{k_{1}\left(k_{1}-k_{2}\right)}, \\
S_{3}(z)=z_{3}+\frac{z_{5} z_{6}}{k_{1}+k_{2}}-\frac{k_{3} z_{5}^{2}}{k_{1}^{2}-k_{2}^{2}}+\frac{k_{3} z_{5}^{2}}{2 k_{1}\left(k_{1}-k_{2}\right)} .
\end{gathered}
$$

Let

$$
S=\left[\begin{array}{ll}
S_{4}(z) & S_{3}(z)
\end{array}\right]^{T} .
$$

From (13), it appears clearly that if the state variables belong to the 2-dimensional manifold

$$
M_{S}=\left\{z \in R^{4} \mid S_{4}(z)=0, S_{3}(z)=0\right\},
$$

then the whole state $z$ tends to the origin, since $z_{5}$ and $z_{6}$ decay exponentially to zero. Furthermore, this manifold is invariant under the linear state feedback (9), as it is shown in the following result.

Proposition 4. Consider the following functions:

$$
\begin{aligned}
& S_{4}(z)=z_{4}-\frac{k_{3} z_{5}}{k_{2}\left(k_{1}-k_{2}\right)}+\frac{z_{6}}{k_{2}}+\frac{k_{3} z_{5}}{k_{1}\left(k_{1}-k_{2}\right)}, \\
& S_{3}(z)=z_{3}+\frac{z_{5} z_{6}}{k_{1}+k_{2}}-\frac{k_{3} z_{5}^{2}}{k_{1}^{2}-k_{2}^{2}}+\frac{k_{3} z_{5}^{2}}{2 k_{1}\left(k_{1}-k_{2}\right)} .
\end{aligned}
$$

Then $M_{S}=\left\{z \in R^{4} \mid S_{4}(z)=0, S_{3}(z)=0\right\}$ is an invariant manifold for the closed-loop system (8)-(10).

Proof. Denote vector fields of system (10) under the linear state feedback (9)

$$
f=z_{5} z_{6} \frac{\partial}{\partial z_{3}}+z_{6} \frac{\partial}{\partial z_{4}}-k_{1} z_{5} \frac{\partial}{\partial z_{5}}-\left(k_{2} z_{6}+k_{3} z_{5}\right) \frac{\partial}{\partial z_{6}} .
$$

Evaluating the Lie derivatives of along the vector fields (16) yields

$$
\begin{aligned}
& L_{f} S_{4}(z) \\
& =\frac{\partial S_{4}}{\partial z} f(z) \\
& =\left[\begin{array}{c}
0 \\
1 \\
-\frac{k_{3}}{k_{2}\left(k_{1}-k_{2}\right)}+\frac{k_{3}}{k_{1}\left(k_{1}-k_{2}\right)} \\
\frac{k_{2}}{2}
\end{array}\right]^{T} \\
& \times\left[\begin{array}{c}
z_{5} z_{6} \\
z_{6} \\
-k_{1} z_{5} \\
-k_{2} z_{6}-k_{3} z_{5}
\end{array}\right] \\
& =z_{6}+\frac{k_{1} k_{3} z_{5}}{k_{2}\left(k_{1}-k_{2}\right)}-\frac{k_{3} z_{5}}{\left(k_{1}-k_{2}\right)}-z_{6}-\frac{k_{3}}{k_{2}} z_{5} \\
& =0 \text {, } \\
& L_{f} S_{3}(z) \\
& =\frac{\partial S_{3}}{\partial z} f(z) \\
& =\left[\frac{\partial S_{3}}{\partial z_{3}}, \frac{\partial S_{3}}{\partial z_{4}}, \frac{\partial S_{3}}{\partial z_{5}}, \frac{\partial S_{3}}{\partial z_{6}}\right] f(z) \\
& =\left[1,0, \frac{z_{6}}{k_{1}+k_{2}}-\frac{2 k_{3} z_{5}}{k_{1}^{2}-k_{2}^{2}}+\frac{2 k_{3} z_{5}}{2 k_{1}\left(k_{1}-k_{2}\right)}, \frac{z_{5}}{k_{1}+k_{2}}\right] \\
& \times\left[z_{5} z_{6}, z_{6},-k_{1} z_{5},-\left(k_{2} z_{6}+k_{3} z_{5}\right)\right]^{T} \\
& =0 \text {. }
\end{aligned}
$$

It appears clearly that the state variables $M_{S}$ belong to the 2dimensional invariant manifold.

Hence, we can construct the invariant manifold of the system (10) as $M_{S}$, which has the following characters:

(1) $z=0 \in M_{s}$;

(2) to stabilize system (8) exponentially, it suffices to bring the state variables $\left[\begin{array}{llll}z_{5} & z_{6} & z_{4} & z_{3}\end{array}\right]$ into $M_{S}$ by an additional state feedback, namely, $w_{1}, w_{2}$.

Now it appears clearly that if the initial conditions are locating in the invariant manifold $M_{S}$, the system variables decay exponentially to zero in terms of linear feedback control. If the initial conditions locate outside of $M_{S}$, in order to stabilize the system, we should force the system variables into $M_{S}$ firstly and then use the feedback control to stabilize the system.

In this paper, an MPC method is proposed for system (8) with the initial conditions out of the manifolds $M_{S}$. Consider an initial state $\left[\begin{array}{llll}z_{5} & z_{6} & z_{4} & z_{3}\end{array}\right]$ and a control horizon of $T$. At initial time, let the manifolds $M_{S}$ be the terminal constraint set. The first objective of the proposed algorithm is to use a T-step control horizon to steer the terminal set-valued state prediction $[z(t+T)]$ into the terminal constraint set $M_{S}$. The detail solution can be achieved by the minimization problem 
in Section 3.2. Secondly, use the feedback control to stabilize the system to the origin.

3.2. Design of MPC Controller. In this subsection we focus our objective on the determination of the first term to make the MPC controller steer the state into the terminal manifold $M_{S}$, asymptotically attractive. Once on it, the whole state $\left[\begin{array}{llll}z_{5} & z_{6} & z_{4} & z_{3}\end{array}\right]$ tends to zero under the residual linear state feedback (9).

MPC is an attractive strategy for systems subject to terminal constraints. Due to the USV systems with terminal constraints, we obtain the control input by minimizing a nominal cost $\left[J\left(z, w_{c}\right)\right]$ over a finite predictive horizon as follows:

$$
J\left(z, w_{c}\right)=\min _{u_{c}} \int_{t}^{t+T} L\left(z, w_{c}\right) d t+W(x(t+T))
$$

s.t.

$$
\begin{gathered}
\dot{z}_{3}=z_{5} z_{6}, \quad \dot{z}_{4}=z_{6}, \\
\dot{z}_{5}=w_{1}, \quad \dot{z}_{6}=w_{2}, \quad z(t+T) \in M_{s},
\end{gathered}
$$

where $T, L\left(z, w_{c}\right)$, and $W(x(t+T))$ denote the time horizon, the running, and terminal costs. $M_{s}$ denotes the terminal constraint set.

Substituting $L\left(z, w_{c}\right)=S^{2}$ by $S=\sqrt{s_{3}^{2}+s_{4}^{2}}$, we can obtain

$$
M_{s}^{2}=S^{2}=\left(z_{3}+\frac{z_{6}}{k_{2}}\right)^{2}+\left(z_{2}+\frac{z_{4} z_{6}}{k_{1}+k_{2}}\right)^{2} \text {. }
$$

Selecting $L\left(z, w_{c}\right)=S^{2}$ and $W=0$ leads to

$$
J\left(z, w_{c}\right)=\min _{w_{c}} \int_{t}^{t+T}\left(\left(z_{3}+\frac{z_{6}}{k_{2}}\right)^{2}+\left(z_{2}+\frac{z_{4} z_{6}}{k_{1}+k_{2}}\right)^{2}\right) d t .
$$

Proposition 5. If the optimization problem in (18) and (19) is feasible, the closed-loop underactuated system (10) with terminal invariant manifolds constraints $M_{s}$ is asymptotically stable in terms of MPC controller.

Proof. Firstly, define the "MPC value function" as $V ;\left(\bar{z}, \bar{w}_{c}\right)$ denotes the optimal solution of (18), $\delta$ denotes the sampling time, and $T$ denotes the predictive and control horizon. There exists a scalar $\varepsilon>0$ such that for each time $t \in[T, \infty)$ and each $z_{t} \in M_{s}$, we can choose a control function $\bar{w}_{c}:[t, t+$ $\varepsilon] \rightarrow R^{2}$, satisfying [33]

$$
\frac{\partial W\left(z_{t}\right)}{\partial z} f\left(z_{t}, \bar{w}_{c}(t)\right) \leq-L\left(z_{t}, \bar{w}_{c}(t)\right)
$$

In sampling time $t_{i}$, the value function for $J\left(t_{i}, z_{t_{i}}, T\right)$ is

$$
V_{t_{i}}=\int_{t_{i}}^{t_{i}+T} L\left(\bar{z}(t), \bar{w}_{c}(t)\right) d t+W\left(\bar{z}\left(t_{i}+T\right)\right) .
$$

Choose sample time $\delta<\varepsilon$ small enough such that extending the process $\left(\bar{z}, \bar{w}_{c}\right)$ to $\left[t_{i}, t_{i}+T+\delta\right], \bar{u}_{c}\left[t_{i}+T, t_{i}+T+\delta\right]$ will satisfy (22). To this control it will correspond to the extended trajectory $\bar{z}\left[t_{i}+T, t_{i}+T+\delta\right]$. The condition (22) guarantees that the extended process $\left(\bar{z}, \bar{w}_{c}\right)$ taken in the interval $\left[t_{i}+\delta, t_{i}+\right.$ $T+\delta$ ] is admissible for problem $J\left(t_{i}+\delta, \bar{z}_{t_{i}+\delta}, T\right)$. However, since this process is not necessarily optimal, we have

$$
\begin{aligned}
V_{t_{i}+\delta}\left(t_{i}+\delta, \bar{z}\left(t_{i}+\delta\right)\right) \leq & \int_{t_{i}+\delta}^{t_{i}+T+\delta} L\left(\bar{z}(t), \bar{w}_{c}(t)\right) d t \\
& +W\left(\bar{z}\left(t_{i}+T+\delta\right)\right) .
\end{aligned}
$$

Hence

$$
\begin{aligned}
V_{t_{i}+\delta}\left(t_{i}\right. & \left.+\delta, \bar{z}\left(t_{i}+\delta\right)\right)-V_{t_{i}}\left(t_{i}, \bar{z}\left(t_{i}\right)\right) \\
\leq & -\int_{t_{i}}^{t_{i}+\delta} L\left(\bar{z}(t), \bar{w}_{c}(t)\right) d t \\
& +\int_{t_{i}+T}^{t_{i}+T+\delta} L\left(\bar{z}(t), \bar{w}_{c}(t)\right) d t \\
& +W\left(\bar{z}\left(t_{i}+T+\delta\right)\right)-W\left(\bar{z}\left(t_{i}+T\right)\right) .
\end{aligned}
$$

Integrating (22), we have

$$
\begin{aligned}
W\left(\bar{z}\left(t_{i}+T+\delta\right)\right)-W\left(\bar{z}\left(t_{i}+T\right)\right) \\
\quad+\int_{t_{i}+T}^{t_{i}+T+\delta} L\left(\bar{z}(t), \bar{w}_{c}(t)\right) d t \leq 0 .
\end{aligned}
$$

Finally, we obtain

$$
\begin{gathered}
V_{t_{i}+\delta}\left(t_{i}+\delta, \bar{z}\left(t_{i}+\delta\right)\right)-V_{t_{i}}\left(t_{i}, \bar{z}\left(t_{i}\right)\right) \\
\leq-\int_{t_{i}}^{t_{i}+\delta} L\left(\bar{z}(t), \bar{w}_{c}(t)\right) d t
\end{gathered}
$$

Due to $L\left(z, w_{c}\right) \geq 0$, we know the value function is decreasing on each interval $\left[t_{i}, t_{i}+\delta\right]$ for any $i$ and the function $V$ is smaller at $t_{i+1}$ than at $t_{i}$. Hence the close-loop system (8) is asymptotically stable.

The stabilizing properties of this approach can be confirmed by the existence of an admissible solution to the openloop optimization (21) at initial time $t$, and so stability is guaranteed provided that $M_{s}$ is reachable in time. Then the condition $L\left(z, w_{c}\right)=0$ is guaranteed by using the linear feedback control $w_{c}$. Because $L\left(z, w_{c}\right)=0$, the condition $\dot{W}=0 \leq L\left(z, w_{c}\right)=0$ is satisfied. It is clear that condition (22) is satisfied. (A thorough discussion of the previous problems can be found in [33].)

The approach was first described in [33]. In this case, outside the invariant manifolds centered at the origin, we have to solve the open-loop optimal control problem with (21). Before we reach $M_{s}$, we have a free time problem. After the set $M_{s}$ is reached, we switch to a linear stabilizing feedback controller for the linearized system. The course of solution is described as follows:

$$
w_{c}=w_{c \mathrm{MPC}}, \quad z \notin M_{s}, \quad w_{c}=w_{c M_{s}}, \quad z \in M_{s},
$$

where $w_{c \text { MPC }}$ denotes the control law by using nonlinear MPC and denotes the linear feedback control law. 


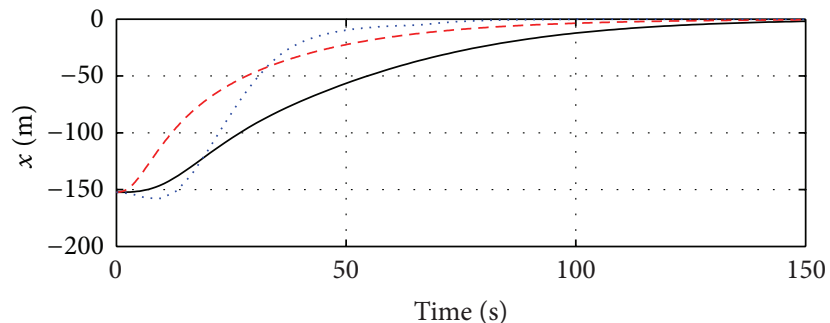

_ Reference [19]: backstepping

- - MPC

Reference [11]: sliding mode

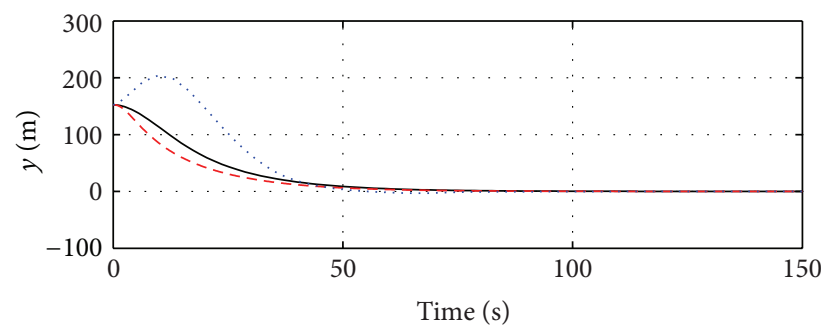

- Reference [19]
- - MPC

Reference [11]

Figure 2: Trajectory of $x$ and $y$.

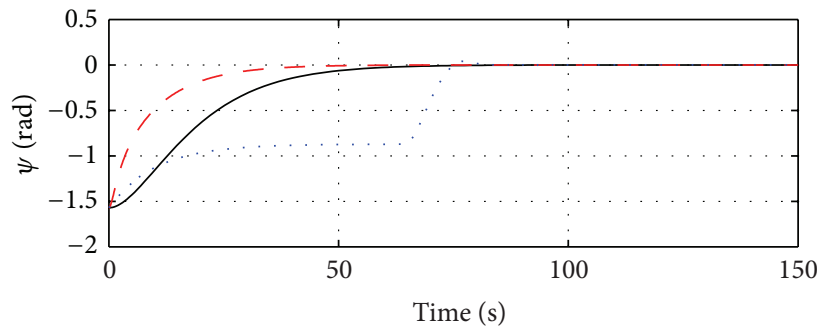

_ Reference [19]

- - MPC

Reference [11]

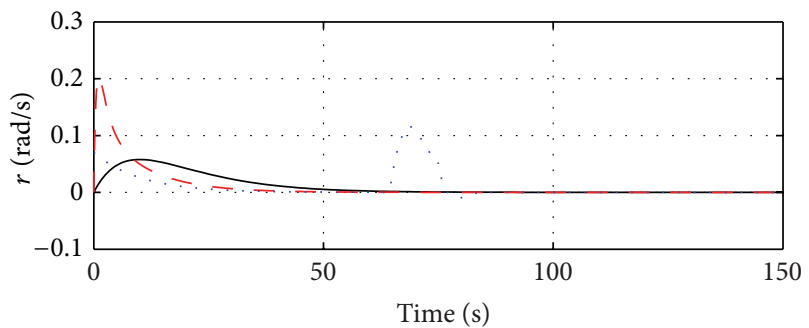

- Reference [19]

- - MPC

Reference [11]

FIgURE 3: Trajectory of $\psi$ and $r$.
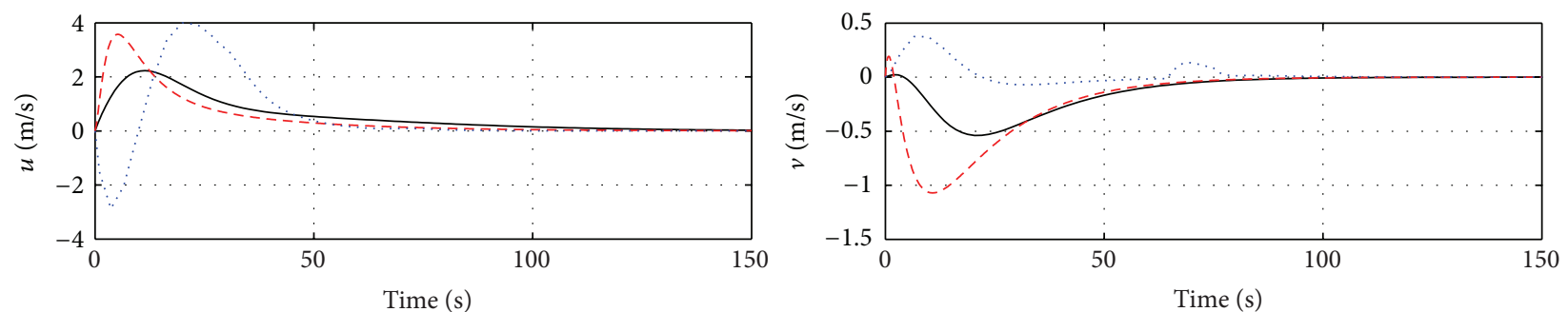

_ Reference [19]

-. MPC

...... Reference [11]

_ Reference [19]

- - MPC

...... Reference [11]

FIGURE 4: Trajectory of $u$ and $v$.
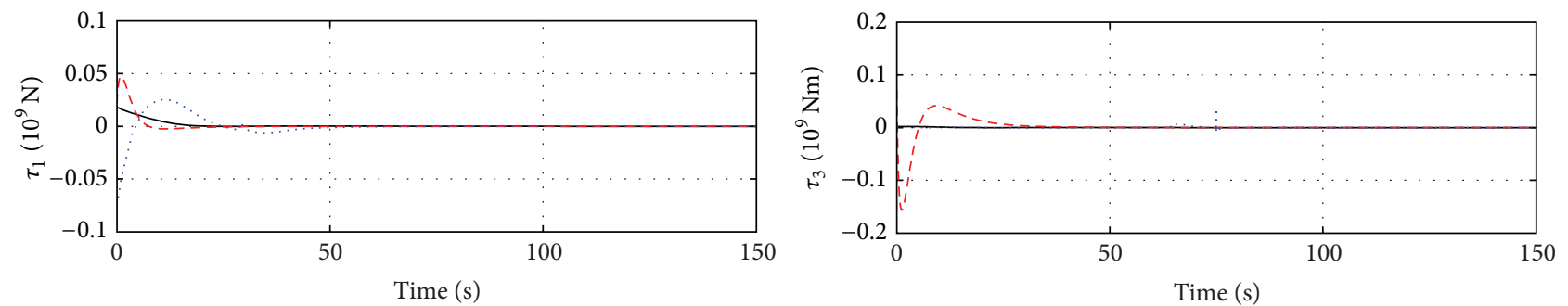

_ Reference [19]: backstepping

- - - MPC

…. Reference [11]: sliding mode

_ Reference [19]: backstepping

- - - MPC

…. Reference [11]: sliding mode

FIGURE 5: Control input. 


\section{Simulation Results}

In [9], an underactuated actual ship named "Northern Clipper" is introduced. Consider the simulation model with parameters as "Northern Clipper" in [9]: $m_{11}=5.312 \times 10^{6} \mathrm{~kg}$, $m_{22}=8.283 \times 10^{6} \mathrm{~kg}, m_{33}=3.745 \times 10^{6} \mathrm{~kg}, d_{11}=5.024 \times$ $10^{4} \mathrm{~kg} / \mathrm{s}, d_{22}=2.722 \times 10^{5} \mathrm{~kg} / \mathrm{s}$, and $d_{33}=1.189 \times 10^{8} \mathrm{~kg} / \mathrm{s}$. Length of "Northern Clipper" $L=76.2 \mathrm{~m}$, and mass $m=$ $4.6 \times 10^{6} \mathrm{~kg}$. In this section, the effectiveness of the proposed MPC control law is verified by following simulation. For the purpose of comparisons, more simulations with sliding mode control approach proposed in [11] and backstepping control proposed in [19] are done to verify the advantage of the MPC method. The control law is selected as Proposition 5, and the initial values are selected as $x(0)=-152.4 \mathrm{~m}, y(0)=$ $-152.4 \mathrm{~m}, \psi(0)=-\pi / 2 \mathrm{rad}, u(0)=v(0)=0 \mathrm{~m} / \mathrm{s}$, and $r=0 \mathrm{rad} / \mathrm{s}$.

The control parameters are selected as $k_{1}=1.2, k_{2}=$ 0.8 , and $k_{3}=1.5$. The performance cost is selected as (21). The terminal manifolds are selected as $M_{s}$. Sampling time is selected as $\delta=0.5 \mathrm{~s}$. Predictive and control horizon are selected as $T=5 \mathrm{~s}$.

Simulation results are shown in the following figures, and the simulation time is set as $150 \mathrm{~s}$. Figure 2 gives the time response of the position $x, y$. Figure 3 gives the time response of the orientation $\psi$ and orientation velocity $r$. Figure 4 gives the time response of the velocities $u, v$. The responses of the control inputs $\tau_{1}$ and $\tau_{3}$ are shown in Figure 5.

Figures $2-5$ show that the three control laws all asymptotically stabilize the underactuated surface vessel to the zero origin. Since the MPC control law guarantees a faster convergence rate of the simulation system, the MPC method may be superior to the other two. Furthermore, Figure 5 shows that surge control force $\tau_{1}$ given by MPC is always positive. Compared with reverse thrust for an actual ship, positive thrust is easier to achieve in practice. Therefore, the MPC approach gets a more favorable control effect.

\section{Conclusion}

This paper proposes the stabilization approach for underactuated surface vessels with only a surge force and a yaw moment. The invariant manifolds constraints are studied, and stability theory of MPC controller is further developed, which is applied to the stabilization control of underactuated surface vessel. For the stabilization control of underactuated surface vessel, a nonlinear MPC control law with terminal invariant manifolds constraints is designed through coordinate transformation and state feedback transformation based on diffeomorphism and Lyapunov stability theory. The simulation results show that the proposed control law can effectively deal with the problem of stabilization control of underactuated surface vessel.

It should be noted that the controller proposed in this paper is developed with constant parameters. In the future work, efforts will be made to design the data-driven controller with uncertain parameters to reduce the effect of noisy data and computational complexity.

\section{Acknowledgments}

The authors are grateful to their colleagues from the College of Information and Telecommunication and the College of Automation in Harbin Engineering University for providing helpful comments. This work is partially supported by the National Natural Science Foundation of China (61201410 and 51379044), Fundamental Research Funds for the Central Universities (HEUCF130804), and Heilongjiang Province Natural Science Foundation Projects (F200916).

\section{References}

[1] R. Hedjar, "Adaptive neural network model predictive control," International Journal of Innovative Computing, Information and Control, vol. 9, no. 3, pp. 1245-1257, 2013.

[2] V. Vesely, D. Rosinova, and T. Nguyen Quang, "Networked output feedback robust predictive controller design," International Journal of Innovative Computing, Information and Control, vol. 9, no. 10, pp. 3941-3953, 2013.

[3] S. Bououden, M. Chadli, F. Allouani, and S. Filali, "A new approach for fuzzy predictive adaptive controller design using particle swarm optimization algorithm," International Journal of Innovative Computing, Information and Control, vol. 9, no. 9, pp. 3741-3758, 2013.

[4] V. Vesely and J. Osusky, "Robust multivariable generalized predictive control design," International Journal of Innovative Computing, Information and Control, vol. 9, no. 8, pp. 3377-3390, 2013.

[5] X. Su, P. Shi, L. Wu, and Y. -D. Song, "A novel control design on discrete-time takagi-sugeno fuzzy systems with time-varying delays," IEEE Transactions on Fuzzy Systems, vol. 20, no. 6, pp. 655-671, 2013.

[6] X. Su, X. Yang, P. Shi, and L. Wu, "Fuzzy control of nonlinear electromagnetic suspension systems," Mechatronics.

[7] L. Wu, W. X. Zheng, and H. Gao, "Dissipativity-based sliding mode control of switched stochastic systems," IEEE Transactions on Automatic Control, vol. 58, no. 3, pp. 785-793, 2013.

[8] L. Wu, X. Su, and P. Shi, "Output feedback control of markovian jump repeated scalar nonlinear systems," IEEE Transactions on Automatic Control.

[9] T. I. Fossen, Marine Control Systems, Marine Cybernetics, Trondheim, Norway, 2002.

[10] K. D. Do and J. Pan, "Robust path-following of underactuated ships: theory and experiments on a model ship," Ocean Engineering, vol. 33, no. 10, pp. 1354-1372, 2006.

[11] J. Cheng, J. Yi, and D. Zhao, "Stabilization of an underactuated surface vessel via discontinuous control," in Proceedings of the American Control Conference, pp. 206-211, New York, NY, USA, July 2007.

[12] K. Y. Pettersen and H. Nijmeijer, "Global practical stabilization and tracking for an underactuated ship - a combined averaging and backsteppihg approach," Modeling, Identification and Control, vol. 20, no. 4, pp. 189-199, 1999.

[13] K. Y. Pettersen and H. Nijmeijer, "Tracking control of an underactuated surface vessel," in Proceedings of the 37th IEEE Conference on Decision and Control, pp. 4561-4566, Tampa, Fla, USA, December 1998.

[14] E. Lefeber, Tracking control of nonlinear mechanical systems [Ph.D. thesis], Department of Mechanical Engineering, Twente University, Twente, The Netherlands, 2000. 
[15] E. Lefeber, K. Y. Pettersen, and H. Nijmeijer, "Tracking control of an underactuated ship," IEEE Transactions on Control Systems Technology, vol. 11, no. 1, pp. 52-61, 2003.

[16] Z. P. Jiang and H. Nijmeijer, "A recursive technique for tracking control of nonholonomic systems in chained form," IEEE Transactions on Automatic Control, vol. 44, no. 2, pp. 265-279, 1999.

[17] K. Y. Pettersen and H. Nijmeijer, "Underactuated ship tracking control: theory and experiments," International Journal of Control, vol. 74, no. 14, pp. 1435-1446, 2001.

[18] Z. P. Jiang, "Global tracking control of underactuated ships by Lyapunovs direct method," Automatica, vol. 38, no. 2, pp. 301309, 2002.

[19] J. Ghommam, F. Mnif, A. Benali, and N. Derbel, "Asymptotic backstepping stabilization of an underactuated surface vessel," IEEE Transactions on Control Systems Technology, vol. 14, no. 6, pp. 1150-1157, 2006.

[20] Z. Liu, R. Yu, and Q. Zhu, "Comments on asymptotic backstepping stabilization of an underactuated surface vessel," IEEE Transactions on Control Systems Technology, vol. 20, no. 1, pp. 286288, 2012.

[21] Li Zhen, Path following with roll constraints for marine surface vessels in wave fields [Ph.D. thesis], University of Michigan, Ann Arbor, Mich, USA, 2009.

[22] X. F. Wang, T. S. Li, Z. J. Zou, and W. L. Luo, "Nonlinear model predictive controller design for path following of underactuated ships," Journal of Ship Mechanics, vol. 14, no. 3, pp. 217-227, 2010.

[23] S. Yin, S. X. Ding, A. Haghani, H. Hao, and P. Zhang, "A comparison study of basic data-driven fault diagnosis and process monitoring methods on the benchmark Tennessee Eastman process," Journal of Process Control, vol. 22, no. 9, pp. 1567-1581, 2012.

[24] S. Yin, S. Ding, and H. Luo, "Real-time implementation of fault tolerant control system with performance optimization," IEEE Transactions on Industrial Electronics, vol. 61, no. 5, pp. 24022411, 2013.

[25] S. Yin, S. Ding, A. Haghani, and H. Hao, "Data-driven monitoring for stochastic systems and its application on batch process," International Journal of Systems Science, vol. 44, no. 7, pp. 13661376, 2013.

[26] S. Yin, X. Yang, and H. Karimi, "Data-driven adaptive observer for fault diagnosis," Mathematical Problems in Engineering, vol. 2012, Article ID 832836, 21 pages, 2012.

[27] T. I. Fossen, Guidance and Control of Ocean Vehicles, Wiley, New York, NY, USA, 1994.

[28] K. Y. Wichlund, O. J. Sordalen, and O. Egeland, "Control properties of underactuated vehicles," in Proceedings of the IEEE International Conference on Robotics and Automation, vol. 2, pp. 2009-2014, Nagoya, Japan, May 1995.

[29] C. I. Byrnes and A. Isidori, "On the attitude stabilization of rigid spacecraft,” Automatica, vol. 27, no. 1, pp. 87-95, 1991.

[30] K. Y. Pettersen and O. Egeland, "Exponential stabilization of an nderactuated surface vessel," in Proceedings of the 35th IEEE Conference on Decision and Control, pp. 967-972, Kobe, Japan, 1996.

[31] R. W. Brockett, "Asymptotic stability and feedback stabilization," in Proceedings of Conference Progress in Mathematics, vol. 27, pp. 181-208, Boston, Mass, USA, 1983.

[32] F. Mazenc, K. Y. Pettersen, and H. Nijmeijer, "Global uniform asymptotic stabilization of an underactuated surface vessel," in Proceedings of the 41st IEEE Conference on Decision and Control (CDC '02), pp. 510-515, Las Vegas, Nev, USA, December 2002.
[33] F. A. C. C. Fontes, "A general framework to design stabilizing nonlinear model predictive controllers," Systems and Control Letters, vol. 42, no. 2, pp. 127-143, 2001. 


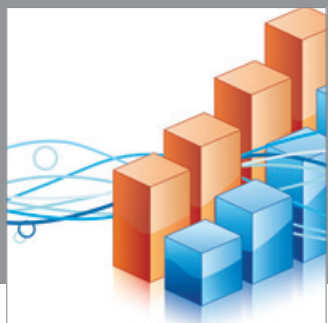

Advances in

Operations Research

mansans

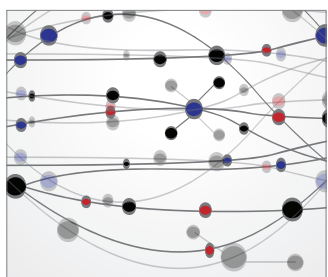

The Scientific World Journal
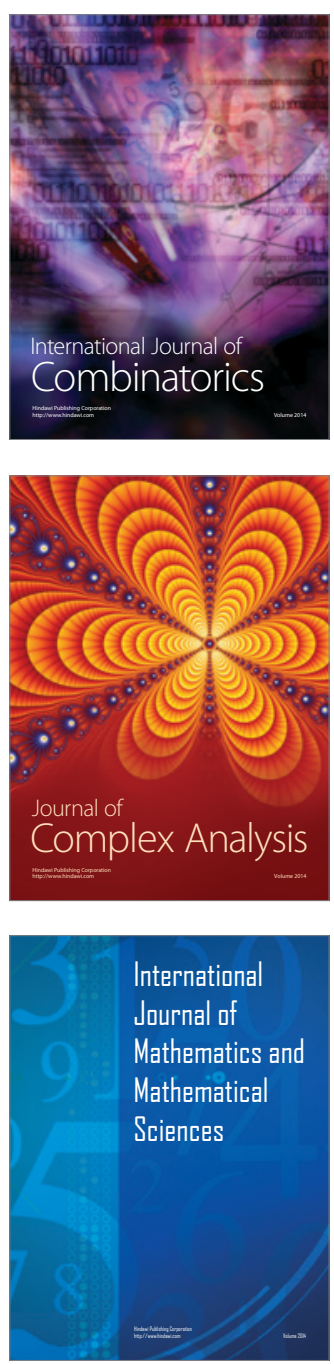
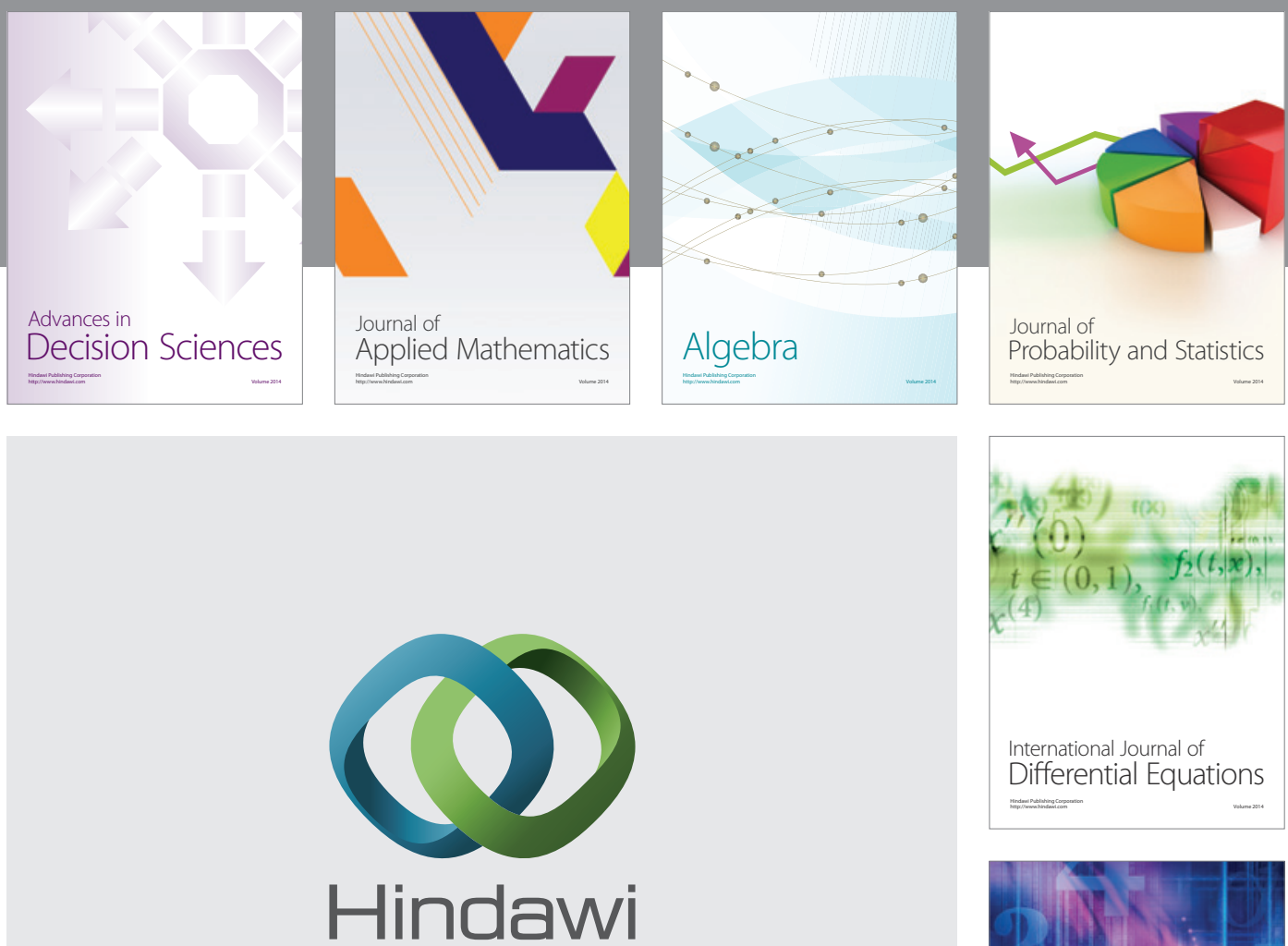

Submit your manuscripts at http://www.hindawi.com
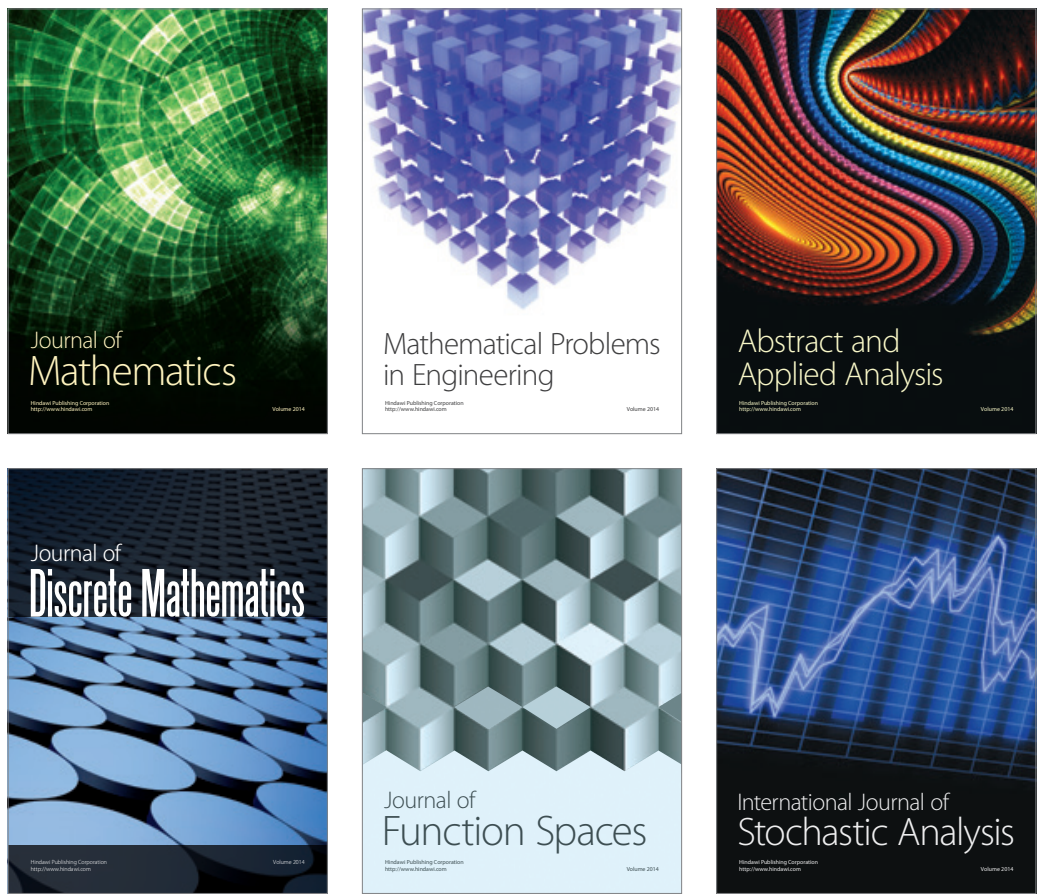

Journal of

Function Spaces

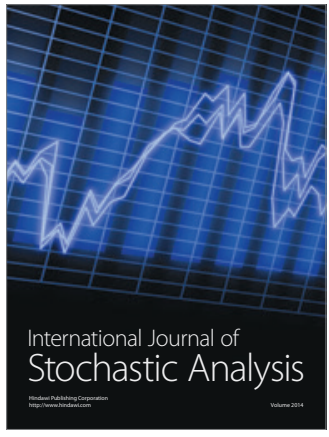

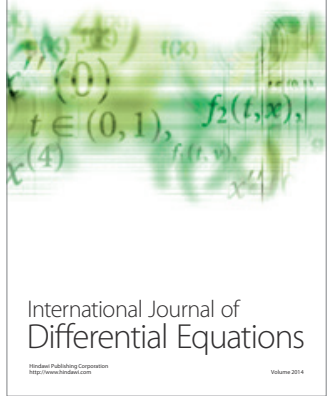
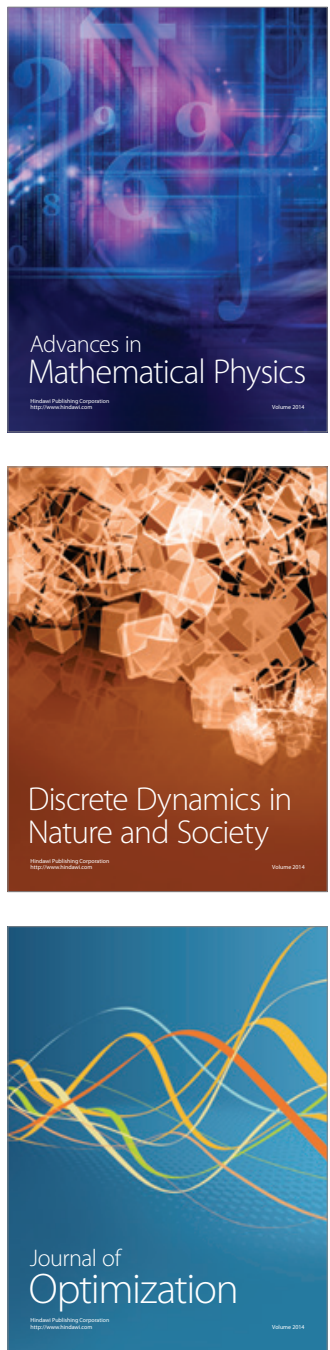